\title{
Maternal micronutrient consumption periconceptionally and during pregnancy: a prospective cohort study
}

\author{
Michelle Livock ${ }^{1,2, *}$, Peter J Anderson 3,4 , Sharon Lewis ${ }^{2,4}$, Stephen Bowden ${ }^{1}$, \\ Evelyne Muggli ${ }^{2,4}$ and Jane Halliday ${ }^{2,4}$ \\ 'Melbourne School of Psychological Sciences, The University of Melbourne, 12th Floor Redmond Barry Building, \\ Parkville, VIC 3010, Australia: 2Public Health Genetics, Murdoch Childrens Research Institute, Royal Children's Hospital, \\ Parkville, Victoria, Australia: ${ }^{3}$ Clinical Sciences, Murdoch Childrens Research Institute, Royal Children's Hospital, \\ Parkville, Victoria, Australia: ${ }^{4}$ Department of Paediatrics, The University of Melbourne, Parkville, Victoria, Australia
}

Submitted 18 December 2015: Final revision received 28 June 2016: Accepted 29 June 2016: First published online 3 August 2016

\begin{abstract}
Objective: To examine overall micronutrient intake periconceptionally and throughout pregnancy in a population-based cohort of Australian women.

Design: In a prospective cohort study, micronutrient dosages were extracted from self-reported maternal supplement use, recorded pre-conception, and for each trimester of pregnancy. A food frequency scale (DQESv2) captured usual maternal diet for gestational weeks 14-26. The influence of sociodemographic and lifestyle factors associated with supplement use was examined using logistic regression, and changes in micronutrient intakes prior to and throughout pregnancy were assessed using repeated-measures ANOVA analyses.

Setting: Metropolitan hospital sites in Melbourne, Australia.

Subjects: Women with a viable singleton pregnancy were recruited at less than 19 weeks' gestation ( $n$ 2146).

Results: Compared with non-users, women using supplements during pregnancy were more likely to have planned their pregnancy, be $>25$ years old, primiparous, Caucasian, non-smokers, have a tertiary education and be consuming a folate-rich diet. Intakes of folate, Fe and $\mathrm{Zn}$ were significantly lower in the periconceptional period, compared with other periods $(P<0 \cdot 001)$. Intakes below Recommended Daily Intake levels were common both periconceptionally and throughout pregnancy, with 19-46\% of women not meeting the Recommended Daily Intake for folate, $68-82 \%$ for $\mathrm{Fe}$ and $17-36 \%$ for $\mathrm{Zn}$. Conversely, 15-19\% of women consumed beyond the recommended Upper Limit for folate and 11-24\% for Fe. Conclusions: The study highlights the need for improved public health education on nutritional needs during pregnancy, especially among women with lower educational achievements and income.
\end{abstract}

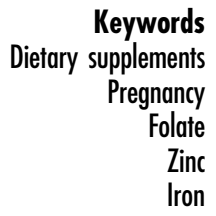

Maternal nutritional status during pregnancy is a key factor in the health and development of the offspring, especially in fetal brain development ${ }^{(1)}$. Of particular importance during pregnancy are methyl-donor nutrients such as folate and vitamin $\mathrm{B}_{12}$, which are considered to be positively associated with brain growth and cognitive development in the offspring ${ }^{(2,3)}$. Folate reduces the risk of neural tube defects in the fetus when consumed in the periconceptional period $^{(4)}$, a finding which has led to the fortification of grain with folic acid in many countries ${ }^{(5)}$. $\mathrm{Zn}$ and $\mathrm{Fe}$ have also been implicated in fetal brain development, with in utero deficiencies associated with higher likelihood of preterm birth and poorer attention, learning and memory ${ }^{(6-8)}$. Research also suggests that vitamin D is crucial for fetal development.
Gestational vitamin D deficiency has been linked to the development of neurological disorders such as autism ${ }^{(9)}$ and, more recently, with language impairment in child$\operatorname{hood}^{(10)}$. An association also exists between vitamin D deficiency during the latter stages of pregnancy and impaired immune functioning and rickets in the offspring $^{(11,12)}$.

Given the heightened nutritional requirements during pregnancy ${ }^{(1,13)}$, some women are at risk for suboptimal intake when relying on diet alone ${ }^{(14,15)}$. To address the perceived gap between diet and recommended nutrient status, consumption of dietary supplements during pregnancy has become increasingly popular, with their prevalence of use ranging from 81 to $97 \%$ in developed nations ${ }^{(14,16-18)}$. Some studies have characterised the types 
of supplements that women take during their pregnancy ${ }^{(14,16,19)}$ and others have examined the nutritional intake of pregnant women through diet ${ }^{(15)}$. Little is known, however, about the total amount of key micronutrients consumed during pregnancy when considering the combined contribution of supplementation and diet. There is also little information on the total nutritional intake of women periconceptionally and in very early pregnancy.

Understanding micronutrient consumption patterns throughout pregnancy and during critical periods of fetal development has important implications for public health policies around maternal nutrition in pregnancy. As such, the objective of the present study was to describe total micronutrient consumption through both diet and supplement use in a population-based prospective cohort of pregnant women.

Specifically, we aimed to: (i) describe supplement use in the three months prior to and throughout pregnancy; (ii) describe the socio-economic, demographic and lifestyle factors associated with supplement consumption; and (iii) using dietary and supplemental intake values, compare with the Australian national pregnancy guidelines ${ }^{(20)}$ the total quantity of key micronutrients consumed both prior to and during pregnancy.

\section{Method}

\section{Study population}

The present study is nested within the AQUA (Asking QUestions about Alcohol) study ${ }^{(21)}$. Women were recruited during early pregnancy at one of seven maternity hospitals in Melbourne, Australia, between July 2011 and July 2012. These sites were chosen based on birth numbers, model of booking in for pregnancy and because of strong management support, thus maximising the feasibility of the project. To be included, women were required to have a viable singleton pregnancy, be less than 19 weeks' gestation at the time of recruitment, at least 16 years of age, fluent in English and able to provide informed consent ( $n$ 2146). The study protocol provides a detailed description of the population of pregnant women involved in $\mathrm{AQUA}^{(21)}$.

\section{Data collection}

Prenatal data collection was administered via three selfcompleted questionnaires covering five time points: Questionnaire 1 , completed at recruitment ( $<19$ weeks' gestation), collected information for three months prior to conception (3MP), after conception but before pregnancy awareness (PPA) and after pregnancy awareness until 13 weeks' gestation (T1; $n$ 2146); Questionnaire 2, completed at 26-28 weeks' gestation, covered the second trimester (T2; $n$ 1715, 79.9\%); and Questionnaire 3, at 36 weeks' gestation, covered the third trimester (T3; $n$ 1571, 73.2\%).
Women recorded their use of dietary supplements for each time point. A check list of nine common vitamins and minerals was provided. These included multivitamins, vitamin $\mathrm{A}$, vitamin $\mathrm{D}$, vitamin $\mathrm{E}$, zinc, iron, folic acid, antacids and fish oil, with the option to provide an 'other' response. Women also recorded their use of up to three specific supplements, including the brand and type of supplement, quantity consumed and frequency of use. The nutrient values of these supplements were gathered from product descriptions provided by the manufacturer or supplier, either on the homepage on the Internet or via personal communication.

To calculate the nutrient composition of the dietary supplements, a database was created containing a collated list of reported supplements, with approximately 400 unique entries. Median values were used when detailed nutritional information on a particular supplement was not available (3\% of participants) and when a supplement type was recorded without a corresponding brand (2-14\% of participants).

Information on maternal diet was collected in Questionnaire 2 through a modified FFQ (Dietary Questionnaire for Epidemiological Studies, Version 2 (DQESv2); Cancer Council Victoria, 1996). The DQESv2 is a measure of usual dietary intake and has been validated for use in large-scale epidemiological studies ${ }^{(22)}$. The questionnaire incorporates cereal foods, sweets, snacks, dairy products, meats, fish, fruit and vegetables. Participants were asked to rate seventy-four specific food types on a 10-point frequency scale ranging from 'never' to multiple times per month, week or day. Global questions regarding quantity and type of hot meals, fruit, vegetables and dairy products typically consumed were also asked, and were used to scale responses. The dietary information collected in Questionnaire 2 has been used as a proxy for participants' usual diet prior to and throughout pregnancy.

Analysis of the FFQ was undertaken by the Cancer Council Victoria. This analysis excluded information on vitamin $\mathrm{B}_{12}$ and vitamin $\mathrm{D}$ intake from food ${ }^{(23)}$. Further, while the Australian Government introduced mandatory fortification of bread flour with folic acid in 2009, it was not possible to modify the calculation of folate intake accordingly ${ }^{(23)}$.

\section{Demographic and lifestyle information}

Detailed data on obstetric history, maternal social environment and broader lifestyle factors were also collected at each time point ${ }^{(21)}$. Factors included in the current analysis were maternal age in years $(<25 ; 25-29 ; 30-34 ; 35-40 ;>40)$, ethnicity (Caucasian; Asian; Other), pre-pregnancy BMI, parity (0 previous children; 1 previous child; $>1$ previous children), pregnancy planning (planned; unplanned), educational attainment (secondary schooling; trade/diploma; tertiary education), annual household income in \$AU (<30 000; 30000-69000; 70000-100000; >100000), and maternal use of tobacco and alcohol (yes; no). 


\section{Statistical approach}

Statistical analyses were conducted using the statistical software package STATA 13.0. Multiple logistic regression was used to assess the independent sociodemographic and lifestyle factors associated with overall supplement use and use of general multivitamins, pregnancy multivitamins, folate-specific supplements and vitamin D supplements. Odds ratios and 95\% confidence intervals were calculated.

To assess differences in micronutrient intake over the five time points (from 3MP to T3), one-way repeated-measures ANOVA were conducted. A Greenhouse-Geisser correction was employed where $\varepsilon<0.75$ and a Huynh-Feldt correction where $\varepsilon>0.75$. $P<0.05$ was considered significant in all statistical tests.

Australian Nutrient Reference Values (national dietary guidelines) for pregnancy ${ }^{(20)}$ were used to evaluate nutrient intake among our participants. The current Recommended Daily Intake (RDI) value for folate during pregnancy is $600 \mu \mathrm{g} / \mathrm{d}$ (from combined food and supplemental sources). Typically, women are advised to take a $400 \mu \mathrm{g}$ folic acid supplement daily in the periconceptional and early pregnancy periods, in addition to consuming folate-rich foods. This recommendation is endorsed by both the US Institute of Medicine ${ }^{(24)}$ and Food Standards Australia New Zealand ${ }^{(25)}$. The RDI for $\mathrm{Fe}$ and $\mathrm{Zn}$ intake during pregnancy is $27 \mathrm{mg} / \mathrm{d}$ and $11 \mathrm{mg} / \mathrm{d}$, respectively ${ }^{(20)}$.

\section{Results}

\section{Supplement use prior to and during pregnancy}

Table 1 shows the distribution of the number and type of supplements consumed at each time point. Sixty-four per cent of women were taking a supplement in the periconceptional and pregnancy pre-awareness (3MP and PPA) periods, rising to $88-91 \%$ in T1 to T3. Among supplement users, multivitamins (either general or pregnancy-specific) were the most commonly consumed supplements at all stages of pregnancy. Approximately $40 \%$ of women from the total sample consumed a folic acid preparation (pregnancy multivitamin, folate-specific supplement and/or folate/iodine supplement) periconceptionally, with this figure rising to $62 \%$ after pregnancy awareness. There was a steady increase in vitamin D supplementation during pregnancy, and the proportion of women using Fe supplements almost tripled from early pregnancy (T1) to T3.

\section{Demographic, socio-economic and lifestyle factors associated with supplement use}

Overall supplement use

Fig. 1(a) and 1(b) show OR and 95\% CI for variables associated with supplement users, compared with non-users, in a multivariate model. Results are separated according to gestational timing. See the online supplementary material, Table S1 for full univariate and multivariate analysis information. When adjusted for all other variables, those positively associated with supplement use were pregnancy planning (associated with use pre-conception and T1), consumption of a folate-rich diet in pregnancy (pre-conception, and trimester one prior to pregnancy awareness) and lower pre-pregnancy BMI (T2 and T3).

Significant negative predictors of supplement use, after adjusting for covariates, included Asian ethnicity (negatively associated with use pre-conception and in T1), 'other' ethnicity (trimester one prior to pregnancy awareness), maternal age $<25$ years (pre-conception, and T1 and T3) or 25-29 years (pre-conception), and having one previous child (pre-conception and T2) or multiple previous children (pre-conception, trimester one, prior to pregnancy awareness, and T2). Additional negative predictors of supplement use included alcohol consumption in pregnancy (negatively associated with use in trimester one, prior to pregnancy awareness) and smoking (pre-conception and trimester one, prior to pregnancy awareness). Finally, annual household income below \$AU 100000 (pre-conception and trimester one, prior to pregnancy awareness) and maternal education to secondary school (pre-conception and T1) or trade/diploma level (pre-conception, and T1 and T3) were also negatively associated with supplement use.

\section{Use of specific supplement types}

Variables associated with use of pregnancy-specific multivitamins, general multivitamins, folic acid-specific supplements and vitamin D supplements were also examined in a multivariate model. Tables $\mathrm{S} 2-\mathrm{S} 5$ in the online supplementary material show OR, 95\% CI and significance levels for each supplement type across the five time periods.

For pregnancy-specific multivitamins, pregnancy planning was a significant positive predictor of use (associated with use pre-conception and trimester one, prior to pregnancy awareness), as was alcohol consumption (T2 and T3). Significant negative predictors of use included annual household income $<$ \$AU 30000 (T1, T2 and T3), annual household income of \$AU 30 000-69000 (trimester one, post pregnancy awareness), education to secondary school level (pre-conception), Asian (preconception and T1, T2 and T3) and 'other' ethnicity (trimester one, post pregnancy awareness), and increasing parity (T3).

For general multivitamins, pregnancy planning was negatively associated with use pre-conception and in trimester one, prior to pregnancy awareness. Significant positive predictors of general multivitamin use included education to secondary school level (associated with use pre-conception), Asian ethnicity (T1, T2 and T3), and annual household income of $<\$$ AU 30000 (trimester one, post pregnancy awareness, and T2) and \$AU 300000 69000 (trimester one, post pregnancy awareness). 
Table 1 Distribution of number and type of supplements consumed across five time points in a population-based prospective cohort of women with a viable singleton pregnancy ( $n$ 2146), Melbourne, Australia, July 2011-July 2012

\begin{tabular}{|c|c|c|c|c|c|c|c|c|c|c|c|c|c|c|c|}
\hline & \multicolumn{3}{|c|}{$3 \mathrm{MP}(n 2146)$} & \multicolumn{3}{|c|}{ PPA $(n$ 2146) } & \multicolumn{3}{|c|}{ T1 (n 2146) } & \multicolumn{3}{|c|}{$\mathrm{T} 2(n$ 1715) } & \multicolumn{3}{|c|}{ T3 $(n$ 1571) } \\
\hline & \multicolumn{2}{|c|}{$\%$} & $n$ & \multicolumn{2}{|c|}{$\%$} & $n$ & \multicolumn{2}{|c|}{$\%$} & $n$ & \multicolumn{2}{|c|}{$\%$} & $n$ & \multicolumn{2}{|c|}{$\%$} & $n$ \\
\hline Taking a supplement & \multicolumn{2}{|c|}{64} & 1379 & \multicolumn{2}{|c|}{64} & 1382 & \multicolumn{2}{|c|}{91} & 1952 & \multicolumn{2}{|c|}{88} & 1505 & \multicolumn{2}{|c|}{91} & 1434 \\
\hline Nil & \multicolumn{2}{|c|}{36} & 767 & \multicolumn{2}{|c|}{36} & 764 & \multicolumn{2}{|c|}{9} & 194 & \multicolumn{2}{|c|}{12} & 210 & \multicolumn{2}{|c|}{9} & 136 \\
\hline One & \multicolumn{2}{|c|}{37} & 787 & \multicolumn{2}{|c|}{37} & 791 & \multicolumn{2}{|c|}{45} & 962 & \multicolumn{2}{|c|}{33} & 567 & \multicolumn{2}{|c|}{32} & 497 \\
\hline Two & \multicolumn{2}{|c|}{18} & 385 & \multirow{2}{*}{\multicolumn{2}{|c|}{$\begin{array}{c}20 \\
8\end{array}$}} & 428 & & & 695 & \multirow{2}{*}{\multicolumn{2}{|c|}{$\begin{array}{l}35 \\
19\end{array}$}} & 608 & \multirow{2}{*}{\multicolumn{2}{|c|}{$\begin{array}{l}34 \\
25\end{array}$}} & 539 \\
\hline Three or more & 10 & & 207 & & & 163 & \multicolumn{2}{|c|}{14} & 295 & & & 330 & & & 398 \\
\hline Supplement types* & SU & TS & $n$ & SU & TS & $n$ & SU & TS & $n$ & SU & TS & $n$ & SU & TS & $n$ \\
\hline General multivitamin & 35 & 22 & 477 & 34 & 22 & 466 & 34 & 31 & 657 & 31 & 27 & 461 & 39 & 35 & 557 \\
\hline Pregnancy/conception multivitamin & 39 & 25 & 533 & 40 & 26 & 556 & 52 & 47 & 1014 & 58 & 51 & 873 & 50 & 46 & 717 \\
\hline B vitamin multivitamin & 1.5 & 1 & 22 & 1 & 1 & 19 & 1 & 1 & 17 & $<1$ & 1 & 10 & $<1$ & $<1$ & 7 \\
\hline Folate and lodine & 2.5 & 2 & 36 & 3 & 2 & 39 & 2 & 2 & 39 & 1.5 & 1 & 23 & 1 & 1 & 14 \\
\hline Folate & 24 & 15 & 329 & 21 & 14 & 291 & 17 & 15 & 326 & 8 & 7 & 124 & 5 & 4 & 68 \\
\hline Zinc & 1 & 1 & 18 & 1 & 1 & 15 & $<1$ & $<1$ & 9 & $<1$ & 1 & 10 & $<1$ & 1 & 9 \\
\hline Iron & 5 & 3 & 72 & 5 & 3 & 71 & 5 & 4 & 92 & 7 & 6 & 105 & 14 & 13 & 206 \\
\hline Vitamin $B_{12}$ & $<1$ & $<1$ & 5 & $<1$ & $<1$ & 7 & $<1$ & $<1$ & 8 & $<1$ & $<1$ & 7 & 1 & 1 & 14 \\
\hline Vitamin D & 18.5 & 12 & 254 & 21 & 14 & 295 & 35 & 32 & 688 & 52 & 45 & 777 & 53 & 48 & 761 \\
\hline Fish/cod liver oils & 13 & 9 & 186 & 12 & 8 & 171 & 7 & 6 & 135 & 8 & 7 & 119 & 7 & 7 & 107 \\
\hline Calcium & 1 & 1 & 11 & 1 & 1 & 13 & 1.5 & 1 & 28 & 2 & 1 & 25 & 2 & 2 & 26 \\
\hline Vitamin D and calcium & 1 & 1 & 18 & 1 & 1 & 18 & 2 & 2 & 33 & 3 & 3 & 45 & 4 & 3 & 54 \\
\hline Herbal & 5 & 3 & 65 & 3 & 2 & 44 & 1.5 & 1 & 28 & 1.5 & 1 & 22 & 4 & 3 & 53 \\
\hline Other & 8 & 5 & 108 & 7 & 5 & 102 & 6 & 5 & 118 & 8 & 7 & 122 & 8 & 7 & 117 \\
\hline Any folic acid preparation $†$ & 62 & 40 & 853 & 62 & 40 & 858 & 68 & 62 & 1320 & 66 & 58 & 991 & 54 & 50 & 780 \\
\hline
\end{tabular}

3MP, three months prior to conception; PPA, after conception but prior to pregnancy awareness; T1, from awareness to gestational week 13; T2, second trimester; T3, third trimester; SU, proportion of supplement users (\%), TS, proportion of total sample (\%).

${ }^{*}$ Categories are not mutually exclusive, as some women are consuming two or more supplement types.

†Includes single or combined use of a pregnancy multivitamin, folic acid-specific supplement and folic acid/iodine supplement.

Pregnancy planning was a positive predictor for periconceptional use of folic acid supplements (preconception and trimester one, prior to pregnancy awareness). Annual household income of $\$ A U$ 70000100000 was a negative predictor of folic acid supplement use pre-conception, compared with households earning $>$ \$AU 100000 per annum.

For vitamin D-specific supplements, Asian (T1 and T3) and 'other' ethnicity (pre-conception and trimester one, prior to pregnancy awareness) were significant positive predictors of use, as was increasing BMI (T2 and T3) and maternal age 25-29 years (T2 and T3). Significant negative predictors included alcohol consumption (negatively associated with vitamin D use pre-conception and in T1, T2 and T3) and maternal education to secondary school level (pre-conception and trimester one, post pregnancy awareness).

\section{Total intake of folate (dietary and supplemental) prior to and during pregnancy}

A one-way repeated-measures ANOVA showed that women's total combined dietary and supplemental intake of folate differed significantly across time points $(F(2 \cdot 96$, $5065.73)=50.27, P<0.001$; data not tabulated). In the periconceptional period almost half of women were consuming below the RDI for folate (Fig. 2). During this time the average total folate intake (3MP: 660 (SD 625) $\mu \mathrm{g} / \mathrm{d}$; PPA: 650 (SD 588) $\mu \mathrm{g} / \mathrm{d}$; data not tabulated) was significantly lower compared with intake following pregnancy awareness (T1: $840 \quad$ (sD 672) $\mu \mathrm{g} / \mathrm{d}, \quad P<0.001$; data not tabulated) and in T2 (760 (sD 604) $\mu \mathrm{g} / \mathrm{d}, P<0.001$; data not tabulated). Women were most likely to overconsume folate in T1, following pregnancy recognition, and average total folate intake was also significantly higher directly after recognition compared with intake in $\mathrm{T} 2(P<0.001$; data not tabulated) and T3 (690 (sD 583) $\mu \mathrm{g} / \mathrm{d}, P<0 \cdot 001$; data not tabulated).

The proportion of total folate intake from supplement use differed substantially across intake categories, with about $80 \%$ of total folate for overconsumers coming from supplements, in contrast to $14-36 \%$ for those with total folate consumption below the RDI (Fig. 2).

\section{Total intake of iron (dietary and supplemental) prior to and during pregnancy}

Total average $\mathrm{Fe}$ intake also differed significantly at different stages of the periconceptional and pregnancy periods $(F(2 \cdot 89, \quad 4932.60)=89.01, \quad P<0.001 ; \quad$ data not tabulated). In the 3MP (24.11 (sD 26.93) $\mathrm{mg} / \mathrm{d}$; data not tabulated) and PPA periods (23.22 (sD 25.06) mg/d; data not tabulated), average total $\mathrm{Fe}$ intake was significantly lower than in the remainder of pregnancy (TI, post awareness: 30.47 (sD 29.66) mg/d, $P<0.001$; T2: 33.33 (sD $34.42) \mathrm{mg} / \mathrm{d}, \quad P<0.001$; T3: 37.36 (sD 41.33 ) $\mathrm{mg} / \mathrm{d}$, $P<0.001$; data not tabulated). Relatively few women were meeting the RDI for $\mathrm{Fe}$, with most participants either 
(a)
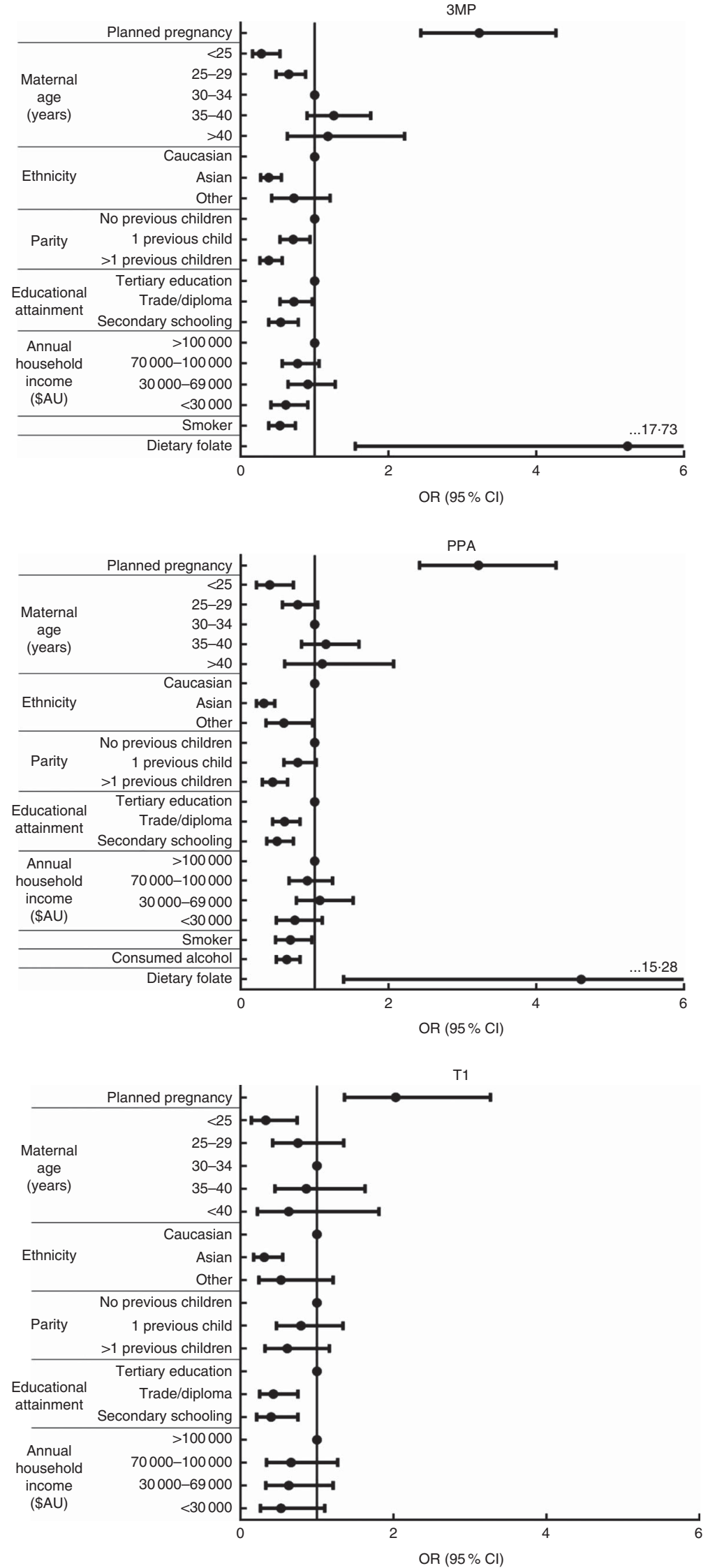

Fig. 1 (Continued on following page) 
(b)
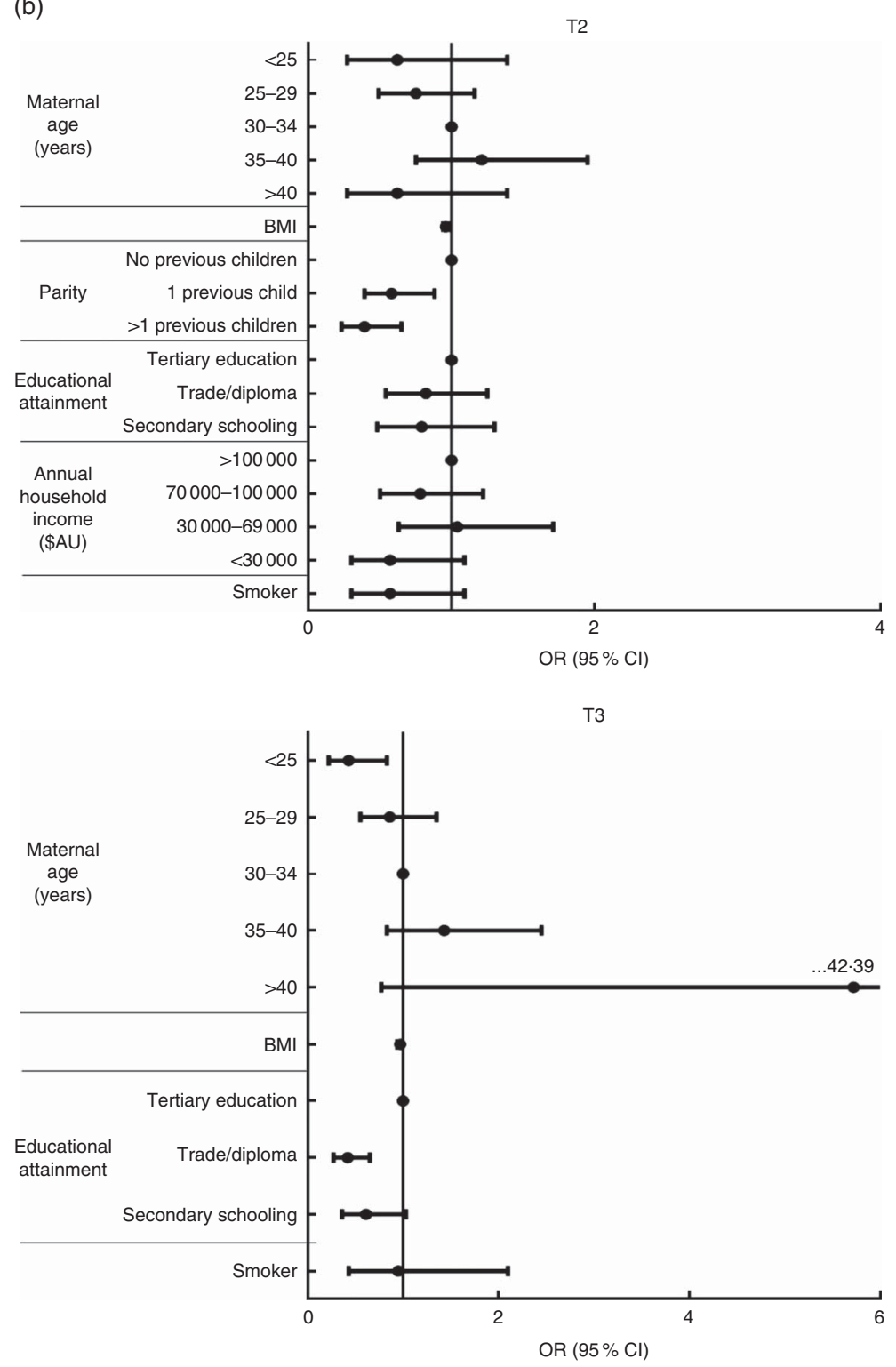

Fig. 1 (Continued from previous page) Multivariate model of variables associated with overall supplement use in (a) early pregnancy (3MP, three months prior to conception; PPA, after conception but prior to pregnancy awareness; T1, from awareness to gestational week 13) and (b) mid to late pregnancy (T2, second trimester; T3, third trimester) among a population-based prospective cohort of women with a viable singleton pregnancy ( $n$ 2146), Melbourne, Australia, July 2011-July 2012. Data are presented as individual odds ratios with $95 \%$ confidence intervals represented by vertical bars. Reference groups: age $=30-34$ years; ethnicity $=$ Caucasian; parity $=0$ previous children; educational attainment $=$ tertiary education; annual household income $=>\$ A U 100000$. Note that all variables are mutually adjusted for each other. Non-significant univariate predictors (3MP: maternal pre-pregnancy BMI, maternal alcohol consumption (yes/no) pre-conception; PPA: maternal pre-pregnancy BMI; T1, post aware: maternal alcohol consumption (yes/no) in T1, maternal smoker status (yes/no) in T1, dietary folate intake, maternal pre-pregnancy BMI; T2: pregnancy planning (yes/no), maternal ethnicity, maternal alcohol consumption (yes/no) in T2, dietary folate intake; T3: pregnancy planning (yes/no), maternal ethnicity, parity, household income, maternal alcohol consumption (yes/no) in T3, dietary folate intake) were excluded from the model

under- or overconsuming this micronutrient (Fig. 3). There was a significant increase in total average Fe intake for each trimester $(P<0 \cdot 01$; data not tabulated), such that almost a quarter of women were consuming beyond the Upper Limit in T2 and T3. Dietary intake of Fe was greatest for those meeting the RDI, with women in this category consuming relatively equal amounts of $\mathrm{Fe}$ through diet and supplementation.

\section{Total intake of zinc (dietary and supplemental) prior to and during pregnancy}

Total average $\mathrm{Zn}$ consumption differed significantly at each time point $(F(3 \cdot 01,5138.99)=96 \cdot 84, P<0 \cdot 001$; data not tabulated). In the periconceptional period over a third of women did not meet the RDI for Zn (Fig. 4) and total average $\mathrm{Zn}$ intake (3MP: 15.20 (sD 9.13) mg/d; PPA: $15 \cdot 47$ (SD 9.23) $\mathrm{mg} / \mathrm{d}$; data not tabulated) was significantly 


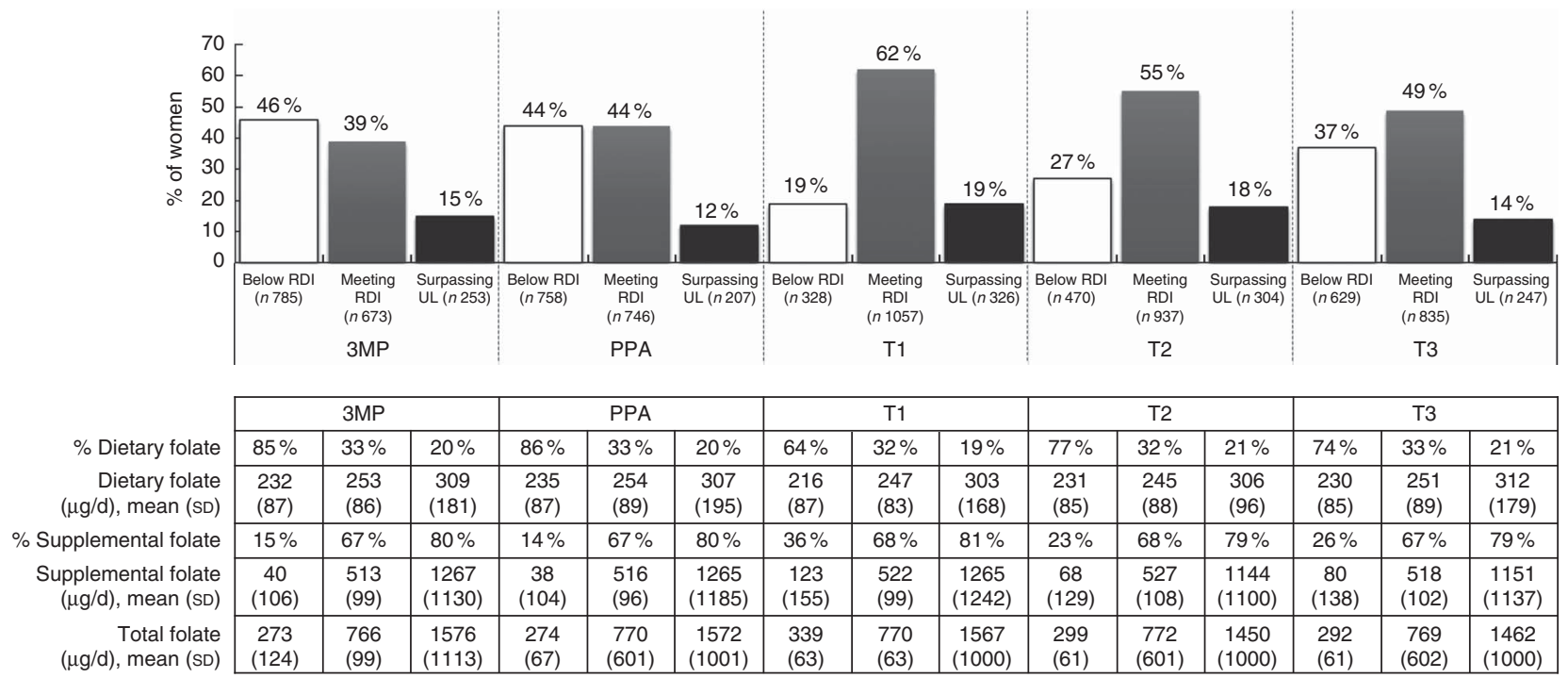

Fig. 2 Total intake of folate from diet and supplementation, according to daily intake category and time period, among a population-based prospective cohort of women with a viable singleton pregnancy ( $n$ 2146), Melbourne, Australia, July 2011-July 2012 (3MP, three months prior to conception; PPA, after conception but prior to pregnancy awareness; T1, from awareness to gestational week 13; T2, second trimester; T3, thirrd trimester; RDI, Recommended Daily Intake; UL, Upper Limit). Intake levels: RDI $=600 \mu \mathrm{g} / \mathrm{d}, \mathrm{UL}=1000 \mu \mathrm{g} / \mathrm{d}$

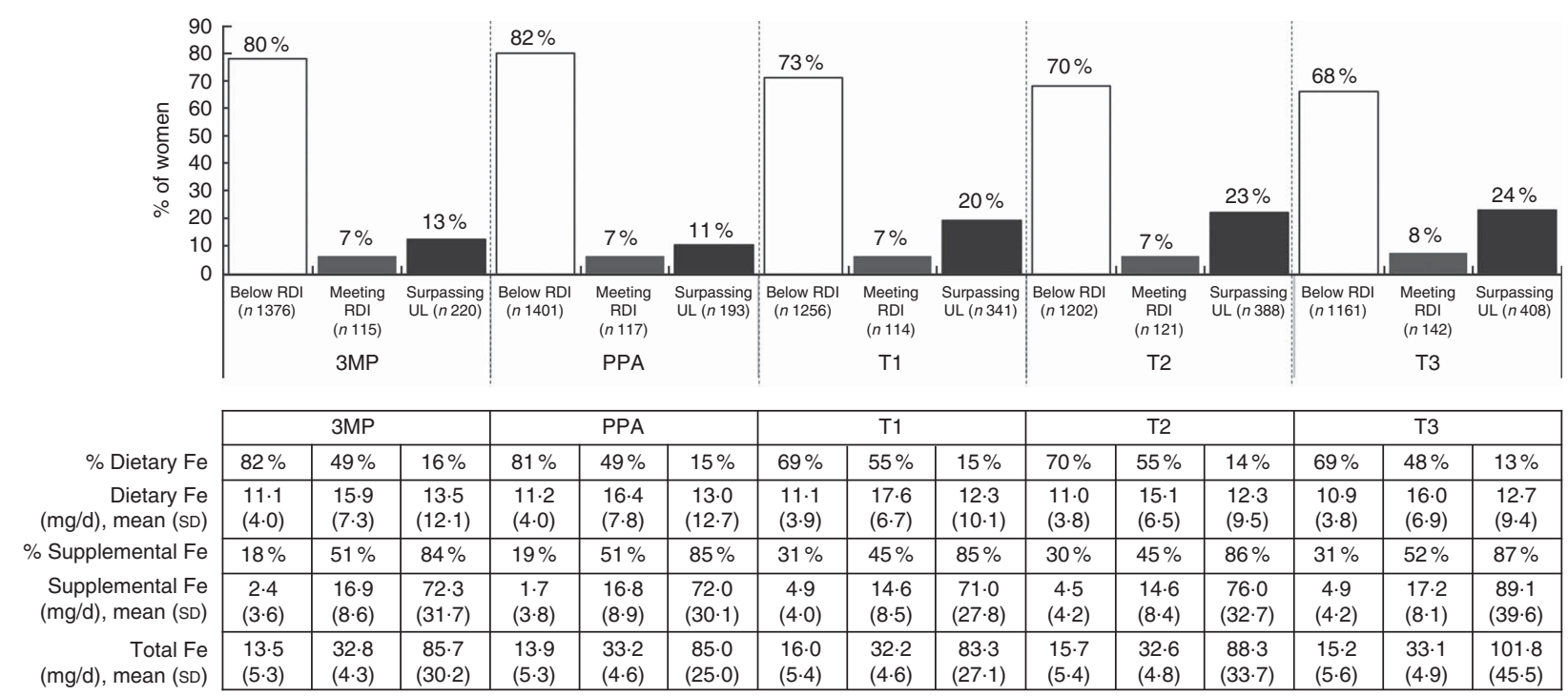

Fig. 3 Total intake of iron from diet and supplementation, according to daily intake category and time period, among a population-based prospective cohort of women with a viable singleton pregnancy ( $n$ 2146), Melbourne, Australia, July 2011-July 2012 (3MP, three months prior to conception; PPA, after conception but prior to pregnancy awareness; T1, from awareness to gestational week 13; T2, second trimester; T3, third trimester; RDI, Recommended Daily Intake; UL, Upper Limit). Intake levels: RDI =27 mg/d; UL =45 mg/d

lower than later in T1, following pregnancy awareness (17.89 (sD 7.66) $\mathrm{mg} / \mathrm{d}, P<0 \cdot 001$; data not tabulated) and in T2 $(17.65(\mathrm{sD} 8 \cdot 18) \mathrm{mg} / \mathrm{d}, P<0 \cdot 001)$ and T3 $(17 \cdot 13(\mathrm{sD}$ 8.37) $\mathrm{mg} / \mathrm{d}, P<0 \cdot 001$; data not tabulated). Total $\mathrm{Zn}$ intake was also significantly higher in T1 post awareness and in T2, compared with T3 $(P<0.001 ; P<0.01$; data not tabulated).

For women meeting or surpassing the RDI, both diet and supplementation contributed to overall intake levels, while for women consuming below the RDI, total intake was predominantly dietary. Overconsumption of $\mathrm{Zn}$ was rare at any time point.

\section{Supplemental intake of vitamin $B_{12}$ and vitamin $D$ prior to and during pregnancy}

Consumption of vitamin $\mathrm{B}_{12}$ and vitamin $\mathrm{D}$ through dietary supplements increased sharply following pregnancy awareness (Table 2). Post awareness, over two-thirds of women were meeting the RDI for vitamin $\mathrm{B}_{12}$, and over 


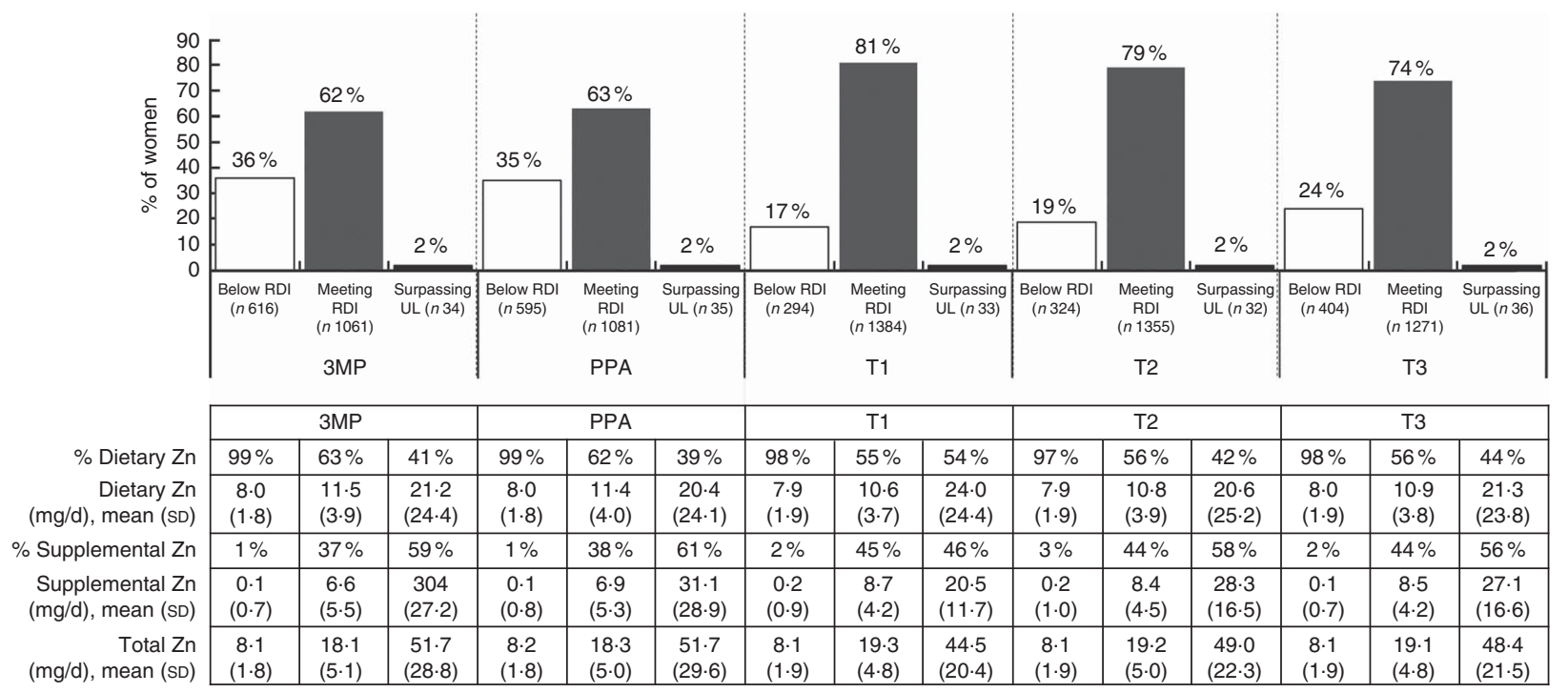

Fig. 4 Total intake of zinc from diet and supplementation, according to daily intake category and time period, among a population-based prospective cohort of women with a viable singleton pregnancy (n2146), Melbourne, Australia, July 2011-July 2012 (3MP, three months prior to conception; PPA, after conception but prior to pregnancy awareness; T1, from awareness to gestational week 13; T2, second trimester; T3, third trimester; RDI, Recommended Daily Intake; UL, Upper Limit). Intake levels: RDI =11 mg/d; UL =35 mg/d

Table 2 Supplemental intake of vitamin $\mathrm{B}_{12}$ and vitamin $\mathrm{D}$, based on supplement users, according to time period, among a population-based prospective cohort of women with a viable singleton pregnancy ( $n$ 2146), Melbourne, Australia, July 2011-July 2012

\begin{tabular}{|c|c|c|c|c|c|c|c|c|c|c|}
\hline & \multicolumn{2}{|c|}{ 3MP } & \multicolumn{2}{|c|}{ PPA } & \multicolumn{2}{|c|}{$\mathrm{T} 1$} & \multicolumn{2}{|c|}{$\mathrm{T} 2$} & \multicolumn{2}{|c|}{ T3 } \\
\hline & $\%$ & $n$ & $\%$ & $n$ & $\%$ & $n$ & $\%$ & $n$ & $\%$ & $n$ \\
\hline Meeting the RDI for vitamin $B_{12}$ & 41 & 890 & 43 & 921 & 68 & 1453 & 65 & 1121 & 68 & 1068 \\
\hline Meeting the Al for vitamin D & 47 & 1014 & 50 & 1064 & 81 & 1736 & 81 & 1394 & 85 & 1335 \\
\hline
\end{tabular}

3MP, three months prior to conception; PPA, after conception but prior to pregnancy awareness; T1, from awareness to gestational week 13; T2, second trimester; T3, third trimester; RDI, Recommended Daily Intake; Al, Adequate Intake (used when RDI cannot be determined).

Intake levels: vitamin $B_{12} \mathrm{RDI}=2.6 \mu \mathrm{g} / \mathrm{d}$; vitamin $\mathrm{D} \mathrm{Al}=5 \mu \mathrm{g} / \mathrm{d}$.

$80 \%$ of women were meeting Adequate Intake levels for vitamin $\mathrm{D}$, through supplementation alone.

\section{Discussion}

In the current large, prospective pregnancy cohort study, intakes of several key micronutrients important for fetal development were examined across five time points. Following pregnancy awareness, supplement use rose to about $90 \%$ and the number of supplements women consumed increased with each trimester. This reflects primarily vitamin $\mathrm{D}$ and $\mathrm{Fe}$ intake, consistent with the increased need for these micronutrients later in pregnancy ${ }^{(20,26,27)}$.

Low intake of dietary supplements during pregnancy is typically seen in those most at risk for nutritional deficiencies (based on sociodemographic characteristics) ${ }^{(18,19)}$, and this was found in the present study with low household income being a negative predictor. Positive predictors of supplement use were pregnancy planning, maternal age $>25$ years, having a first child, Caucasian ethnicity and a tertiary education. In addition, engagement in other positive health behaviours was evident in those using supplements, such as non-smoking, consumption of a folate-rich diet and periconceptional alcohol abstinence.

Women from the lower sociodemographic groups who did consume supplements were more likely to be taking general multivitamins, rather than pregnancy-specific multivitamins, the former being cheaper and having lesser quantities of micronutrients. Further, women meeting the RDI for folate, $\mathrm{Fe}$ and $\mathrm{Zn}$ were typically consuming a higher quantity of these micronutrients through diet, as well as through supplementation, compared with those not meeting the RDI. This indicates that those not meeting dietary requirements have a comparatively nutrient-poor diet which they are less likely to be augmenting with dietary supplements. Public health messages around the importance of nutrition in pregnancy may not be reaching those most at risk and it is plausible that those with a lower income experience barriers to the use of specific-pregnancy preparations.

Women with previous children were also less likely to consume supplements, particularly in the periconceptional period. This lower consumption is unlikely to be due to a lack of awareness around pregnancy nutrition, as multiparous 
women have been shown to have greater knowledge than primiparous women ${ }^{(28)}$. Instead, it may reflect a reduction in pregnancy planning and health proactiveness for subsequent pregnancies. The higher variability in supplement use throughout pregnancy for multiparous women would seem to support this inference.

Ethnicity impacted supplement choice. Caucasian women were more likely to use pregnancy multivitamins at any time prior to or during pregnancy, while women of non-Caucasian ethnicity were more likely to consume general multivitamins and vitamin D-specific supplements. It is possible that health professionals regularly advise women of darker skin and those in ethnic populations requiring the covering of skin with clothing about their increased need for vitamin $\mathrm{D}^{(29)}$.

Somewhat surprisingly, those women continuing to use pregnancy-specific multivitamins into $\mathrm{T} 2$ and $\mathrm{T} 3$ (more likely to be Caucasian, have higher annual household income and education, be primiparous, non-smoking and $>25$ years old) were also more likely to have consumed alcohol in these trimesters. This relationship is consistent with a belief held by some women that low-level consumption of alcohol is associated with low risk, particularly in the context of an otherwise healthy lifestyle ${ }^{(30)}$. While there is some research evidence to support the idea that good maternal nutrition can ameliorate some of the effects of fetal exposure to alcohol ${ }^{(31-33)}$, current medical advice recommends abstinence during pregnancy as the safest policy.

Many women failed to meet the RDI for folate, Fe and Zn, particularly in the critical periconceptional period. Compared with previous studies, we report a slight increase in the proportion of women taking folic acid periconceptionally, from approximately $19-35 \%^{(14,17,28)}$ to $40 \%$. Nevertheless, this indicates that $60 \%$ of women are not consuming a folic acid preparation in this critical time period, despite only $23 \%$ in our sample having an unplanned pregnancy.

Conversely, overconsumption of folate through diet and supplemental sources (beyond $1000 \mu \mathrm{g} / \mathrm{d}$ ) was relatively common, particularly late in T1. There is little consensus on whether nutrient intake beyond a level of sufficiency conveys additional advantage during pregnancy, and recent research has found a link between high doses of folic acid during pregnancy (over $5 \mathrm{mg} / \mathrm{d}$ ) and impaired psychomotor development at 12-23 months of age ${ }^{(34)}$. High intake of folic acid may also lead to adverse neurological outcomes in the fetus by masking a severe vitamin $\mathrm{B}_{12}$ deficiency ${ }^{(20,35,36)}$. Fortunately, when folic acid intake peaked following pregnancy awareness, over two-thirds of women in our sample were meeting the RDI for vitamin $\mathrm{B}_{12}$ through supplementation.

This pattern of folic acid consumption (lowest periconceptionally and increasing late in T1) does not align with knowledge and policy related to primary prevention of neural tube defects. Therefore, while women may be aware that folic acid plays a role in fetal development, and thus begin consumption of this compound following pregnancy recognition, they may be unaware of the importance of timing.

Total combined consumption of dietary and supplemental Fe was polarised, with the majority of women either over- or underconsuming. There were $20-24 \%$ of women consuming above the RDI, which may have been due to specific prescriptions for an identified Fe deficiency that was not captured in the present study. However, increased Fe stores in women not experiencing Fe deficiency have been associated with gestational diabetes and increased oxidative stress during pregnancy ${ }^{(37,38)}$.

Of additional concern was the $70 \%$ of women still not reaching the RDI for $\mathrm{Fe}$ by $\mathrm{T} 3$, despite a progressive rise in average $\mathrm{Fe}$ intake from the periconceptional period. As Fe needs are thought to increase in later pregnancy ${ }^{(26)}$, and animal research has found that Fe deficiency from pre-conception until early in T2 can lead to impairments in fetal neurodevelopment ${ }^{(39)}$, better monitoring and education around Fe status in women of childbearing age is indicated.

\section{Study strengths}

A major strength of the current study was knowledge of women's total nutrient intake, including both diet and supplements. Additionally, by surveying participants at five time points, we were able to measure supplement intake across critical periods of fetal development. To our knowledge, the present study is the first to examine pregnancy micronutrient intake in such detail. A further strength of the research was the large cohort involved and its relatively high retention rate (73\%).

\section{Study limitations}

Measurement error inherent in FFQ must be acknowledged. Despite this, recall-based FFQ have been found to be moderately correlated with biomarker methods ${ }^{(23)}$, particularly for folate, $\mathrm{Zn}$ and $\mathrm{Fe}^{(40)}$. The FFQ was administered in T2, given the prevalence of morning sickness and specific food proclivities in early pregnancy. These data were then used as a proxy for women's usual diet across all time points, potentially reducing the accuracy of dietary intake assigned to other periods. However, evidence suggests that dietary patterns tend to remain stable throughout pregnancy ${ }^{(41,42)}$.

The FFQ we used (DQESv2) does not take into account folic acid fortification of bread flour (introduced in Australia in 2009). However, 27-39\% of Australian households purchase organic bread and breakfast cereals, which are excluded from mandatory fortification requirements ${ }^{(43)}$. Despite this, it should be noted that a $77 \%$ reduction in low serum folate and an $85 \%$ reduction in low red-blood-cell folate in women of childbearing age have been found one year post the introduction of mandatory fortification ${ }^{(44)}$. Further, the mean increase in 
daily dietary folate following mandatory fortification is estimated to be $159 \mu \mathrm{g}$ for women of childbearing age ${ }^{(45)}$. Therefore, it is likely that dietary folate is underestimated in the present study.

Finally, while use of self-report dietary supplement information is highly correlated with biomarker methods $^{(46)}$, use of median nutrient values where detailed nutritional information was not available may impact on the overall accuracy of supplement information. As this occurred for only a small minority of cases, it is unlikely to markedly affect results.

\section{Conclusion}

The present study indicates that over two-thirds of women were consuming supplements periconceptionally and this increased significantly following pregnancy awareness to approximately $90 \%$. When considering the combined contribution of diet and supplementation, the majority of women met the RDI for folate and $\mathrm{Zn}$ throughout pregnancy, but failed to do so periconceptionally. In contrast, a significant minority of women consuming high-dose folic acid supplements exceeded the Upper Level for folate, particularly late in T1 and T2. Intake of Fe remained below the RDI for most women both prior to and throughout pregnancy, with those consuming a low dose or no supplemental Fe least likely to meet the recommended target compared with those taking an Fe-specific supplement.

Supplementation plays a large role in overall micronutrient intake during pregnancy and is necessary for many women to reach RDI levels. The present study highlights the need for improvement in public health education on nutritional needs during pregnancy, particularly among women with lower educational attainment and lower household income.

\section{Acknowledgements}

Acknowledgements: The authors are extremely grateful to all the women and their children for taking part in this study. The authors thank Professor Graham Giles, of the Cancer Epidemiology Centre of The Cancer Council Victoria, for permission to use the Dietary Questionnaire for Epidemiological Studies, Version 2. Financial support: This work was supported by the Australian National Health and Medical Research Council (project grant number 1011070, 2011-2014; Senior Research Fellowship to P.J.A., grant number 1081288; Senior Research Fellowship to J.L.H., grant number 1021252) and the Victorian State Government's Operational Infrastructure Support Program. The study has also received funding from the McCusker Charitable Trust; the Murdoch Childrens Research Institute; the Jack Brockhoff Foundation; and the Foundation for Children.
The funders had no role in the design, analysis or writing of this article. Conflict of interest: The authors report no conflict of interest. Authorship: M.L. performed the analysis and interpretation of results and wrote the manuscript under supervision. P.J.A. and S.L. participated in the planning of the project, assisted with and supervised the statistical analysis and planning and interpretation of results, and critically reviewed the manuscript. S.B. participated in the planning of analyses and critical review of the article. E.M. participated in the planning of the project and assisted with statistical analysis, interpretation and critical review of the manuscript. J.H. conceived the idea for the research project and assisted with planning, statistical analysis, interpretation and critical review of the manuscript. All authors accept responsibility for the paper as published. Ethics of human subject participation: This study was conducted according to the guidelines laid down in the Declaration of Helsinki and all procedures involving human subjects/patients were approved by the Eastern Health Research and Ethics Committee (E54/1011) and the Human Research Ethics Committees of Mercy Health (R11/14), Monash Health (11071B), the Royal Women's Hospital (11/20) and the Royal Children's Hospital (31055A). The latter also approved all follow-up of the children (31055C/ D). Written informed consent was obtained from all subjects/patients.

\section{Supplementary material}

To view supplementary material for this article, please visit http://dx.doi.org/10.1017/S1368980016002019

\section{References}

1. Nyardi A, Li J, Hickling S et al. (2013) The role of nutrition in children's neurocognitive development, from pregnancy through childhood. Front Hum Neurosci 7, 97.

2. Cusick S \& Georgieff M (2012) Nutrient supplementation and neurodevelopment: timing is key. Arch Paediatr Adolesc Med 166, 481-482.

3. Villamor E, Rifas-Shiman S, Gilman M et al. (2012) Maternal intake of methyl-donor nutrients and child cognition at 3 years of age. Paediatr Perinat Epidemiol 26, 328-335.

4. Lumley J, Watson L, Watson M et al. (2001) Periconceptional supplementation with folate and/or multivitamins to prevent neural tube defects. Cochrane Database Syst Rev 3, CD001056.

5. Food Fortification Initiative (2015) Country Profile. http:// www.ffinetwork.org/country_profiles/index.php (accessed August 2015).

6. Uriu-Adams J \& Keen C (2010) Zinc and reproduction: effects of zinc deficiency on prenatal and early postnatal development. Birth Defects Res 89, 313-325.

7. Szajewska H, Ruszczynski M \& Chmielewska A (2010) Effects of iron supplementation in nonanemic pregnant women, infants, and young children on the mental performance and psychomotor development of children: a systematic review of randomized controlled trials. Am J Clin Nutr 91, 1684-1690.

8. Hermoso M, Vucic V, Vollhardt C et al. (2011) The effect of iron on cognitive development and function in infants, children and adolescents: a systematic review. Ann Nutr Metab 59, 154-165. 
9. Cannell J (2008) Autism and vitamin D. Med Hypotheses 70, 750-759.

10. Whitehouse A, Holt B, Serralha M et al. (2012) Maternal serum vitamin D levels during pregnancy and offspring neurocognitive development. Pediatrics 129, 485-493.

11. Brown A, Dusso A \& Slatopolsky E (1999) Vitamin D. Am J Physiol 277, F157-F175.

12. Thandrayen K \& Pettifor J (2010) Maternal vitamin D status: implications for the development of infantile nutritional rickets. Endocrinol Metab Clin North Am 39, 303-320.

13. Picciano M (2003) Pregnancy and lactation: physiological adjustments, nutritional requirements and the role of dietary supplements. J Nutr 133, issue 6, 1997S-2002S.

14. Haugen M, Brantsaeter A, Alexander J et al. (2008) Dietary supplements contribute substantially to the total nutrient intake in pregnancy Norwegian women. Ann Nutr Metab 52, 272-280.

15. Branstaeter A, Haugen M, Myhre R et al. (2014) Diet matters, particularly in pregnancy - results from MoBa studies of maternal diet and pregnancy outcomes. Norsk Epidemiol 24, 63-77.

16. Gomez M, Field C, Olstad D et al. (2015) Use of micronutrient supplements among pregnant women in Alberta: results from the Alberta Pregnancy Outcomes and Nutrition (APrON) cohort. Matern Child Nutr 11, 497-510.

17. Forster D, Wills G, Denning A et al. (2007) The use of folic acid and other vitamins before and during pregnancy in a group of women in Melbourne, Australia. Midwifery 25, 134-146.

18. Aronsson C, Vehik K, Yang J et al. (2013) Use of dietary supplements in pregnant women in relation to sociodemographic factors - a report from The Environmental Determinants of Diabetes in the Young (TEDDY) study. Public Health Nutr 16, 1390-1402.

19. Pouchieu C, Levy R, Faure C et al. (2013) Socioeconomic, lifestyle and dietary factors associated with dietary supplement use during pregnancy. PLOS ONE 8, e70733.

20. National Health and Medical Research Council (2006) Nutrient Reference Values for Australia and New Zealand, Including Recommended Dietary Intakes. Canberra: Australian Government, Department of Health and Aging.

21. Muggli E, O'Leary C, Forster D et al. (2014) Study protocol: Asking QUestions about Alcohol in pregnancy (AQUA): a longitudinal cohort study of fetal effects of low to moderate alcohol exposure. BMC Pregnancy Childbirth 14, 302-313.

22. Hodge A, Patterson A, Brown W et al. (2000) The Anti Cancer Council of Victoria FFQ: relative validity of nutrient intakes compared with weighed food records in young to middle-aged women in a study of iron supplementation. Aust N Z J Public Health 24, 576-583.

23. Cancer Council Victoria (2015) Dietary Questionnaire for Epidemiological Studies Version 2 (DQESv2); User Information Guide. Melbourne: Cancer Council Victoria.

24. Institute of Medicine (1998) Dietary Reference Intakes for Thiamin, Riboflavin, Niacin, Vitamin $B_{6}$, Folate, Vitamin $B_{12}$, Pantothenic Acid, Biotin, and Choline. Washington, DC: National Academy Press.

25. Food Standards Australia New Zealand (2015) Folic acid/ folate and pregnancy. http://www.foodstandards.gov.au/ consumer/generalissues/pregnancy/folic/Pages/default.aspx (accessed August 2015).

26. O'Brien K, Zavaleta N, Abrams S et al. (2003) Maternal iron status influences iron transfer to the fetus during the third trimester of pregnancy. Am J Clin Nutr 77, 924-930.

27. Specker B (2004) Vitamin D requirements during pregnancy. Am J Clin Nutr 80, 6 Suppl., 1740S-1747S.
28. Watson L, Brown S \& Davey M (2006) Use of periconceptional folic acid supplements in Victoria and New South Wales, Australia. Aust N Z J Public Health 30, 42-49.

29. Williamson C \& Wyness L (2013) Nutritional requirements in pregnancy and use of dietary supplements. Community Pract 86, 44-47.

30. Holland K, McCallum K \& Blood W (2015) Conversations about Alcohol and Pregnancy. Canberra: Foundation for Alcohol Research and Education.

31. Keen C, Uriu-Adams J, Skalny A et al. (2010) The plausibility of maternal nutritional status being a contributing factor to the risk for fetal alcohol spectrum disorders: the potential influence of zinc status as an example. BioFactors 36, 125-135.

32. Rufer E, Tran T, Attridge $M$ et al. (2012) Adequacy of maternal iron status protects against behavioural, neuroanatomical, and growth deficits in fetal alcohol spectrum disorder. PLOS ONE 7, e47499.

33. Coles C, Kable J, Keen et al. (2015) Dose and timing of prenatal alcohol exposure and maternal nutritional supplements: developmental effects on 6 month old infants. Matern Child Health J 19, 2605-2614.

34. Valera-Gran D, Garcia de la Hera M, Navarraete-Munoz E et al. (2014) Folic acid supplements during pregnancy and child psychomotor development after the first year of life. JAMA Pediatr 168, e142611.

35. Choi J, Yates Z, Veysey M et al. (2014) Contemporary issues surrounding folic acid fortification initiatives. Prev Nutr Food Sci 19, 247-260.

36. Hure A, Collins C \& Smith R (2012) A longtitudinal study of maternal folate and vitamin $\mathrm{B}_{12}$ status in pregnancy and postpartum, with the same infant markers at 6 months of age. Matern Child Health J 16, 792-801.

37. Lao T, Chan P \& Tam K (2001) Gestational diabetes mellitus in the last trimester - a feature of maternal iron excess? Diabet Med 18, 218-223.

38. Scholl T (2005) Iron status during pregnancy: setting the stage for mother and infant. Am J Clin Nutr 81, issue 5, 1218S-1222S.

39. Mihaila C, Schramm J, Strathmann F et al. (2011) Identifying a window of vulnerability during fetal development in a maternal iron restriction model. PLOS ONE 6, e17483.

40. Fayet F, Flood V, Petocz P et al. (2011) Relative and biomarker-based validity of a food frequency questionnaire that measures the intakes of vitamin $\mathrm{B}_{12}$, folate, iron, and zinc in young women. Nutr Res 31, 14-20.

41. Sotres-Alvarez D, Herring A \& Siega-Riz A (2013) Latent transition models to study women's changing of dietary patterns from pregnancy to 1 year postpartum. Am J Epidemiol 177, 852-861.

42. Crozier S, Robinson S, Godfrey K et al. (2009) Women's dietary patterns change little from before to during pregnancy. J Nutr 139, 1956-1963.

43. Monk A, Mascitelli B, Lobo A et al. (2012) Australian Organic Market Report. Chermside, Australia: BFA Ltd.

44. Brown R, Langshaw M, Uhr E et al. (2011) The impact of mandatory fortification of flour with folic acid on the blood folate levels of an Australian population. Med J Aust 194, 65-67.

45. Dugbaza J \& Cunningham J (2012) Estimates of total dietary folic acid intake in the Australian population following mandatory folic acid fortification. J Nutr Metab 2012, 492353 .

46. Brantsaeter A, Haugen M, Hagve T et al. (2007) Self-reported dietary supplement use is confirmed by biological markers in the Norwegian Mother and Child Cohort study (MoBa). Ann Nutr Metab 51, 146-154. 